\title{
Evolução Intra-hospitalar e Seguimento Pós-alta de Pacientes Idosos Atendidos com Insuficiência Cardíaca Congestiva na Unidade de Emergência
}

\author{
Humberto Villacorta, Nazareth Rocha, Rosana Cardoso, Sérgio Gaspar, Eduardo Reis Maia, \\ Therezil Bonates, Daniel Kopiler, Hans J. F. Dohmann, Evandro Tinoco Mesquita
}

Rio de Janeiro, RJ

Objetivo - Conhecer a evolução intra-hospitalar $(I H)$ e pós-alta $(P A)$ de uma população predominantemente idosa, com insuficiência cardíaca congestiva (ICC) na unidade de emergência (UE).

Métodos - Durante 11 meses, foram selecionados 57 pacientes consecutivos com ICC, atendidos em EU, com idade média de 69+15 (27 a 94) anos, sendo $39(68,4 \%)$ homens. O diagnóstico de ICC baseou-se nos critérios de Boston. Avaliou-se a evolução IH e PA num período médio

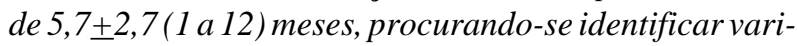
áveis que se correlacionassem com a mortalidade e o mecanismo de morte, avaliado pelo sistema ACME.

Resultados - Oito (14\%) pacientes faleceram na fase IH, sendo 7 por falência circulatória (FC), e 1 em pós-operatório $(P O)$. Durante o seguimento ocorreram $9(18,4 \%)$ óbitos, sendo 5 por FC, 2 mortes súbitas e 2 em PO (troca valvar mitral e ventriculectomia). A sobrevida dos pacientes que tiveram altafoi de $82 \%$ e 66\%, aos 6 meses e 1 ano, respectivamente. Sódio sérico $<135 \mathrm{mEq} / \mathrm{l}(p=0,004) e$ sexo feminino $(p=0,038)$ foram preditores independentes de mortalidade.

Conclusão - Pacientes idosos com ICC atendidos em UE apresentam mortalidade IH e PA elevadas e alta taxa de reinternação hospitalar. A maioria morre por falência circulatória decorrente da progressão da ICC.

Palavras-chave: insuficiência cardíaca congestiva, unidade de emergência, prognóstico

\section{Hospital Outcome and Short-Term Follow-up of PredominatlyElderly Patients Presenting to the Emergency Department with Congestive Heart Failure}

Purpose - To evaluate the in-hospital (IH) outcome and the short-term follow-up of predominantly elderly patients presenting to an emergency room (ER) with congestive heart failure ( $\mathrm{CHF})$.

Methods - In an 11 month period, 57 patients presenting to the ER with CHF were included. Mean age was $69 \pm 15$ years $(27$ to 94$)$ and $39(68,4 \%)$ were male. $\mathrm{CHF}$ diagnosis was based on the Boston criteria. We evaluated IH outcome and prognosis in a mean follow-up of 5,7 $\pm 2,7$ months (1 to 12). In addition, some mortality predictors and mechanisms of death according on the ACME system were identified.

Results - Eight patients (14\%) died in the IH period. Modes of death were circulatory failure $(C F)$ in 7 , and peri-operative $(P O)$ in one (aortic valve replacement). During follow-up 9 deaths ocurred. Five were due to $C F, 2$ were sudden and 2 were $P O$ (mitral valve replacement and ventriculectomy). Six-months and 1-year survival rates of the patients who were discharged were $82 \%$ and $66 \%$, respectively. Sodium lower than $135 \mathrm{mEq} / \mathrm{l}(p=0.004)$ and female gender $(p=0.038)$ were independent predictors of mortality.

Conclusion - Elderly patients with CHF admitted to the ER have high in-hospital and short-term follow up mortalities. The majority die from $C F$ due to worsening heart failure.

Key-words: congestive heart failure, emergency department, prognosis

Arq Bras Cardiol, volume 70 (n 3), 167-171, 1998

Hospital Pró-Cardíaco - Rio de Janeiro

Correspondência: Humberto Villacorta - Hospital Pró-Cardíaco - Unidade de Emergência - Rua Dona Mariana, 219 - 22280-020 - Rio de Janeiro, RJ

Recebido para publicação em 27/10/97

Aceito em 7/1/98
A síndrome de insuficiência cardíaca congestiva (ICC) apresenta prevalência de 1 a $2 \%$ na população geral ${ }^{1-3}$. Em indivíduos com idades entre 65 e 67 anos, esse número eleva-se para $23 \%{ }^{4}$, tornando-se problema sério em idosos. Nos Estados Unidos é a principal causa de internação em 
pessoas com idade $>65$ anos ${ }^{5,6}$. Estima-se que quase $4 \mathrm{mi}-$ lhões de americanos sejam afetados pela doença e cerca de 400.000 novos casos são diagnosticados a cada ano ${ }^{7}$. No Hospital Pró-Cardíaco, a ICC é responsável por 10 a 15\% das internações na sala de emergência ${ }^{8}$, constituindo-se na $3^{a}$ maior causa.

Apesar do arsenal terapêutico, hoje disponível, e dos benefícios alcançados com as drogas vasodilatadoras ${ }^{9-12}$, a mortalidade ainda é elevada, com sobrevida de $25 \%$ em 5 anos ${ }^{13,14}$. Pacientes com ICC atendidos em unidades de emergência (UE) apresentam formas graves da doença e têm sido pouco estudados em nosso meio.

Este estudo visa a avaliar as características clínicas e a evolução intra e extra-hospitalar de uma população predominantemente idosa, atendida com ICC na UE de um centro cardiológico terciário, bem como determinar os mecanismos de morte e fatores prognósticos nesses pacientes.

\section{Métodos}

No período de fevereiro a dezembro/96, foram estudados 57 pacientes consecutivos com diagnóstico de ICC, atendidos na UE de um hospital cardiológico. O diagnóstico, sempre feito por um cardiologista, foi baseado nos critérios de Boston ${ }^{15}$, tendo sido incluído apenas aqueles cuja pontuação permitia um diagnóstico definitivo de ICC. A classe funcional (CF) no momento do atendimento foi estabelecida de acordo com critérios da New York Heart

\begin{tabular}{|ll|}
\hline \multicolumn{1}{|c|}{ Tabela I - Observações relacionadas à morte em pacientes com ICC } \\
segundo o sistema ACME
\end{tabular}

AVC- acidente vascular cerebral; EAP- edema agudo de pulmão; FC- falência circulatória; VE- ventrículo esquerdo; PCR- parada cardiorrespiratória, após

\begin{tabular}{|ll|}
\hline \multicolumn{2}{|c|}{ Tabela II - Características basais dos pacientes } \\
\hline Sexo M/F & $39 / 18$ \\
Idade (anos) & $69 \pm 15$ \\
Idade >65 anos & $40 / 57(70 \%)$ \\
Classe funcional (NYHA) & $3,7 \pm 0,5$ \\
Cardiopatia isquêmica & $34 / 57(60 \%)$ \\
Infarto do miocárdio prévio & $15 / 57(26 \%)$ \\
Revascularização miocárdica prévia & $17 / 57(30 \%)$ \\
Angioplastia coronária prévia & $7 / 57(12 \%)$ \\
Hipertensão arterial sistêmica & $14 / 57(24 \%)$ \\
Diabetes mellitus & $15 / 57(26 \%)$ \\
Valvopatia & $6 / 57(11 \%)$ \\
Cardiomiopatia hipertrófica & $1 / 57(2 \%)$ \\
Fibrilação atrial crônica & $10 / 57(18 \%)$ \\
Insuficiência renal prévia & $4 / 57(7 \%)$ \\
Freqüência cardíaca (batimentos/min) & $102 \pm 22$ \\
Pressão arterial (mmHg) & $96 \pm 12$ \\
Fração de ejeção (\%) & $43 \pm 14$ \\
Fração de encurtamento (\%) & $20 \pm 1$ \\
Sódio sérico (mEq/L) & $134 \pm 7$ \\
Potássio sérico (mmol/L) & $4,5 \pm 0,6$ \\
Cálcio sérico (mmol/L) & $8,5 \pm 0,8$ \\
Magnésio sérico (mg/dL) & $1,9 \pm 0,3$ \\
Hemoglobina (g\%) & $13 \pm 2$ \\
\hline M- masculino; F- feminino; NYHA- classificação da New York Heart \\
Association. & \\
\hline
\end{tabular}

Association (NYHA). Após o diagnóstico clínico da ICC, todos os pacientes foram submetidos a realização de ecocardiograma, para cálculo da fração de ejeção (FE) (volume diastólico final menos volume sistólico final dividido pelo volume diastólico final) e fração de encurtamento (diâmetro diastólico final menos diâmetro sistólico final dividido pelo diâmetro diastólico final). Pacientes com critérios para o diagnóstico de ICC e com FE $\geq 50 \%$ foram considerados como portadores de ICC puramente diastólica. Os critérios de exclusão foram infarto do miocárdio nos últimos 30 dias, angina de peito na admissão, pericardite constritiva, doença pulmonar obstrutiva crônica e neoplasias malignas associadas.

Os pacientes que receberam alta foram seguidos através de contatos telefônicos, por um tempo médio de 5,7 $\underline{2,7}$ meses (variando de um a 12 meses), não ocorrendo perdas. A avaliação das mortes ocorridas nesse período foi realizada através da análise de prontuários médicos - nos casos em que a morte ocorreu durante reinternação em nosso hospital - ou de informações colhidas com familiares e médicos assistentes. A data limite para a avaliação de sobrevida foi 31/1/97. As definições de morte súbita e morte por falência circulatória foram estabelecidas segundo recentes critérios propostos por Narang e col ${ }^{16}$, conhecido como sistema ACME (tab. I), que leva em conta a atividade e local de morte, assim como a causa, modo e eventos a ela associados. As variáveis utilizadas como fatores prognósticos foram aquelas obtidas na admissão do doente na UE.

Variáveis contínuas com distribuição normal foram comparadas através do teste t de Student, para amostras não pareadas. Variáveis discretas foram comparadas utilizando-se o teste do qui-quadrado. As variáveis que se mostraram significativas pela análise univariada foram selecionadas para a análise multivariada. A análise estatística 
foi auxiliada pelos softwares Epiinfo versão 6.03, desenvolvido pela Organização Mundial de Saúde e GB-STAT versão 6.0, com supervisão de um profissional da área. A probabilidade de sobrevida desde a data de inclusão no estudo foi analisada pelo método atuarial. Valor de $\mathrm{p}<0,05$ foi considerado estatisticamente significativo. Os resultados foram expressos em média \pm desvio padrão.

\section{Resultados}

A idade média foi de 69+15 (27 a 94) anos, sendo 39 $(68,4 \%)$ do sexo masculino. Haviam sido internados por ICC previamente $36(63 \%)$ casos e estavam em CF III ou IV no momento da admissão 55 (98,2\%). A FE média determinada pelo ecocardiograma foi de $43 \pm 14 \%$ e a fração de encurtamento de $20 \pm 1 \%$. Nove (16\%) pacientes tinham ICC puramente diastólica. A etiologia da ICC foi variada, sendo isquêmica em $34(60 \%)$ pacientes, hipertensiva em $9(16 \%)$, idiopática em seis (11\%), valvular em seis (11\%), alcóolica em um (2\%) e cardiomiopatia hipertrófica em outro caso. Usavam inibidores da enzima conversora de angiotensina (IECA) $35(61,4 \%)$, digital $30(52,6 \%)$ e diuréticos 29 $(50,8 \%)$. Em 50 pacientes foi possível identificar um fator

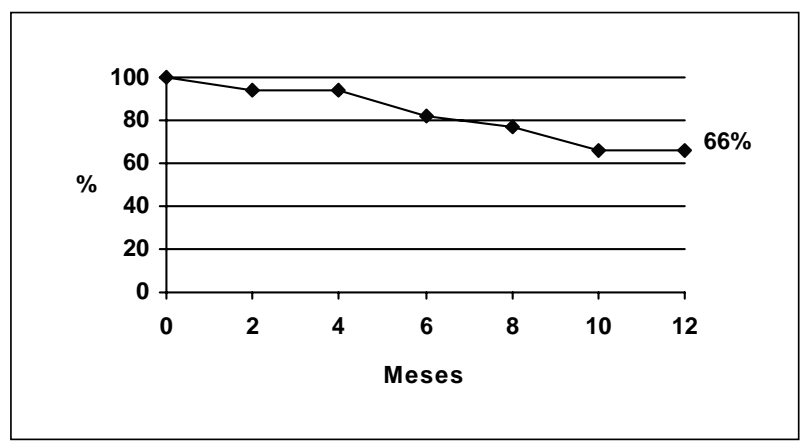

Fig. 1 - Curva de sobrevida pelo método atuarial, dos pacientes que tiveram alta hospitalar precipitante de descompensação, sendo os mais freqüentes hipertensão arterial sistêmica em nove (18\%), abusos na dieta e na ingesta hídrica em sete (14\%), uso irregular de medicação em sete (14\%), infecção respiratória em seis (12\%) e provável isquemia miocárdica (alterações novas no eletrocardiograma) em outros seis pacientes. O tempo médio de internação foi de $9 \pm 8$ dias. Nenhum paciente era vacinado contra infecções por pneumococos ou por vírus da gripe. A tabela II mostra as características basais dos pacientes.

Ocorreram oito (14\%) óbitos na fase intra-hospitalar, todos tendo como causa de morte disfunção miocárdica grave. Quanto ao modo de morte, sete $(87,5 \%)$ foram devidas à falência circulatória (edema agudo de pulmão ou choque cardiogênico) e um (12,5\%) a pós-operatório imediato de troca valvar aórtica. Ocorreu apenas um óbito com menos de $24 \mathrm{~h}$ de internação. Fizeram uso de dobutamina na sala de emergência 34 (60\%) pacientes. O custo hospitalar médio por paciente foi de $\mathrm{R} \$ 12.900,00$, excluindo-se os submetidos a procedimentos cirúrgicos, o valor médio foi de $\mathrm{R} \$ 6.880,00$.

Dos 49 pacientes que sobreviveram à internação, nove $(18,4 \%)$ faleceram durante o seguimento. Oito $(89 \%)$ desses óbitos ocorreram durante reinternações, sendo três (37,5\%) com menos de $24 \mathrm{~h}$ de internação. A causa de morte foi disfunção miocárdica grave em sete $(77,8 \%)$ e arritmia ventricular em dois $(21,2 \%)$. Quanto ao modo de morte, foi súbito em dois $(22,2 \%)$ pacientes, por falência circulatória em cinco $(55,6 \%)$ e peri-operatório em dois, sendo um após troca valvar mitral e outro pós-ventriculectomia. Vinte e cinco pacientes (51\%) tiveram pelo menos uma reinternação. Os procedimentos cardiovasculares ocorridos no seguimento foram troca valvar (dois mitrais e um aórtica) em três (37,5\%) pacientes, ventriculectomia em dois (25\%), revascularização miocárdica em um (12,5\%), aneurismectomia em um $(12,5 \%)$ e implante de marcapasso definitivo em outro paciente.

\begin{tabular}{|c|c|c|c|c|c|c|}
\hline $\mathrm{n}$ & Idade/sexo & Fase do estudo & Local do óbito & Causa & Modo & Evento associado/TEM \\
\hline 1 & $75 / \mathrm{M}$ & Intra-hospitalar & Hospital & DMG & FC & Piora da ICC/1 ano \\
\hline 2 & $49 / \mathrm{F}$ & Intra-hospitalar & Hospital & DMG & Peri-operatória & Troca VAo/7 d \\
\hline 3 & $81 / \mathrm{F}$ & Seguimento & Hospital & DMG & FC & $\mathrm{IAM} / 4 \mathrm{~d}$ \\
\hline 4 & $91 / \mathrm{F}$ & Intra-hospitalar & Hospital & DMG & $\mathrm{FC}$ & $\mathrm{IAM} / 5 \mathrm{~d}$ \\
\hline 5 & $65 / \mathrm{M}$ & Seguimento & Hospital & DMG & Peri-operatória & Aneurismectomia $/<24 \mathrm{~h}$ \\
\hline 6 & 49/M & Seguimento & $\mathrm{Na}$ rua & FV & Súbita & Precordialgia $/<24 \mathrm{~h}$ \\
\hline 7 & 94/M & Intra-hospitalar & Hospital & DMG & FC & Pneumonia/29 d \\
\hline 8 & $93 / \mathrm{F}$ & Seguimento & Hospital & DMG & FC & Piora da ICC $/ 15 \mathrm{~d}$ \\
\hline 9 & $44 / \mathrm{M}$ & Seguimento & Hospital & DMG & Peri-operatória & Ventriculectomia $\quad /<24 \mathrm{~h}$ \\
\hline 10 & $76 / \mathrm{M}$ & Seguimento & Hospital & DMG & FC & Piora da ICC $/ 120 \mathrm{~d}$ \\
\hline 11 & $80 / \mathrm{F}$ & Seguimento & Hospital & DMG & FC & Piora da ICC/90 d \\
\hline 12 & $82 / \mathrm{F}$ & Intra-hospitalar & Hospital & DMG & FC & Piora da ICC/30 d \\
\hline 13 & $65 / \mathrm{F}$ & Seguimento & Hospital & DMG & FC & Piora da ICC/30 d \\
\hline 14 & $79 / \mathrm{M}$ & Seguimento & Hospital & FV & Súbita & Bypass Ao-F/4 d \\
\hline 15 & $85 / \mathrm{F}$ & Intra-hospitalar & Hospital & DMG & FC & $\mathrm{IAM} / 3 \mathrm{~d}$ \\
\hline 16 & $83 / \mathrm{F}$ & Intra-hospitalar & Hospital & DMG & $\mathrm{FC}$ & Piora da ICC/3 d \\
\hline 17 & $86 / \mathrm{M}$ & Intra-hospitalar & Hospital & DMG & $\mathrm{FC}$ & Piora da ICC/3 d \\
\hline
\end{tabular}




\begin{tabular}{|llll|}
\hline \multicolumn{3}{|c|}{ Tabela IV - Comparação global entre sobreviventes (n= 40) e não } \\
sobreviventes (n=17)
\end{tabular}

Os pacientes que faleceram apresentaram em média, menor FE do que os que estavam vivos no final do seguimento, embora sem significância estatística $(34,8 \pm 8$ e $43,8 \pm 1 ; p=0,06)$. A sobrevida destes pacientes aos seis e 12 meses foi $82 \%$ e $66 \%$, respectivamente (fig. 1).

A análise conjunta das evoluções intra-hospitalar e pós-alta mostra que a mortalidade global foi $29,8 \%$ (17 óbitos). A mortalidade foi ligeiramente menor nos pacientes com disfunção diastólica isolada, porém sem atingir significância estatística (22\% vs 31\%; p=0,70). Houve maior mortalidade no sexo feminino ( $50 \%$ contra $20,51 \%$; $\mathrm{p}=0,02)$. A idade média dos pacientes que faleceram foi de $75 \pm 15$ anos contra $66 \pm 14$ dos sobreviventes $(\mathrm{p}=0,04)$. Houve maior mortalidade nos pacientes com sódio plasmático $<135 \mathrm{mEq}$ ( $52 \%$ vs $16,6 \% ; \mathrm{p}=0,01)$. Através de análise multivariada somente o sódio plasmático $(\mathrm{p}=0,004)$ e sexo feminino $(\mathrm{p}=0,038)$ correlacionaram-se de forma independente com a mortalidade. As causas e modos de morte encontram-se na tabela III e a comparação entre sobreviventes e não sobreviventes na tabela IV.

\section{Discussão}

A ICC permanece como um grave problema de saúde pública. Dados atualizados do estudo de Framingham mostram sobrevida média de 1,7 anos para homens e 3,2 anos para mulheres ${ }^{14}$. Além disso, observou-se um aumento na taxa de internações por ICC nas últimas duas décadas ${ }^{17}$. Em nosso estudo, a sobrevida em um ano foi de $66 \%$, menor que a observada em alguns estudos com população semelhante ${ }^{13}$. Atribuímos este achado ao fato de a nossa população ser constituída, em sua maioria, por pacientes idosos - 70\% tinham idade $\geq 65$ anos -, com ICC avançada, a maioria em CF IV. Há, entretanto, alguns trabalhos com taxas de mortalidade próximas à nossa ${ }^{18-20}$. Em um dos raros trabalhos na literatura envolvendo pacientes com ICC na sala de emergên- cia, a mortalidade em seis meses foi de $23 \%$ e a taxa de reinternação foi de $30 \%{ }^{20}$.

Outros fatores, além da idade, poderiam estar contribuindo para alta taxa de reinternação e de mortalidade, como não aderência ao tratamento, fatores socioeconômicos, orientação inadequada quanto a medidas higieno-dietéticas e uso não otimizado dos IECA. Quanto a este último, ressaltamos o fato de que apesar das recomendações existentes na literatura, a maioria dos clínicos ainda utiliza subdoses de IECA $^{21}$. Outro fator freqüentemente esquecido é a prevenção de infecção respiratória através de vacinação contra pneumococos e vírus influenza. Nenhum de nossos pacientes era vacinado e em $12 \%$ deles pneumonia foi a causa de descompensação. Nos pacientes coronários, a falta de pesquisa de viabilidade miocárdica também contribui para a morbimortalidade, pois esses pacientes poderiam obter melhora da função ventricular após algum tipo de revascularização miocárdica ${ }^{22,23}$.

A alta morbimortalidade da ICC resulta em custos elevados. Neste trabalho, o custo médio por paciente foi de $\mathrm{R} \$$ $6.873,00$. Se incluímos os pacientes submetidos a procedimentos cirúrgicos, este valor eleva-se para $\mathrm{R} \$ 12.900,00$. Também chamamos atenção para a alta taxa de reinternação em nossos pacientes, chegando a $51 \%$ em seis meses, contribuindo muito para a elevação dos custos, já que $80 \%$ dos gastos totais com ICC são conseqüentes a hospitalizações ${ }^{24}$. Nos EUA são gastos anualmente, cerca de oito bilhões de dólares, com custo médio de US\$7.000,00 por paciente a cada internação ${ }^{24}$. Isso tem motivado a procura de novas abordagens no manuseio da ICC, visando a reduzir custos. Recentemente, foram criadas as chamadas "clínicas de ICC" formadas por equipe multidisciplinar, composta por médicos, enfermeiros, nutricionistas e fisioterapeutas, tendo-se observado que esse tipo de abordagem reduz a taxa de reinternação, melhora a qualidade de vida e prolonga a sobrevida, ao mesmo tempo em que reduz custos ${ }^{5,25,26}$. Em um estudo, observou-se redução de $44,4 \%$ na taxa de reinternações e de $35,7 \%$ no tempo de hospitalização com mortalidade significativamente menor ${ }^{5}$. Em nosso trabalho, $70,6 \%$ das mortes foi por falência circulatória e somente $11,8 \%$ foram súbitas. Portanto, algumas destas mortes poderiam ter sido evitadas através de educação e vigilância rígida dos pacientes.

Algumas variáveis correlacionaram-se de forma independente com a mortalidade, através de análise multivariada. Sódio plasmático menor que $135 \mathrm{mEq} / \mathrm{L}$ e sexo feminino correlacionaram-se com a mortalidade global. Estas variáveis são descritas, entre outras, como fatores prognósticos na ICC ${ }^{27}$. A presença de hiponatremia identifica um subgrupo de pacientes com hiperativação do sistema renina-angiotensina-aldosterona caracterizando, portanto, estágio mais avançado da doença ${ }^{28}$. Em relação ao sexo, os trabalhos são discordantes, não havendo consenso na literatura $^{29}$.

Neste estudo utilizamos uma nova classificação para mortes em ICC. Esse sistema foi criado em virtude da observação de que as definições de morte súbita e morte por fa- 
lência circulatória encontradas nos estudos da literatura mundial, eram as mais variadas possíveis. Visando uniformizar e facilitar a classificação das mortes, Narang e col ${ }^{16}$ propuseram um sistema que valoriza a atividade e local de morte, bem como causa, modo e eventos a ela associados (sistema ACME), facilitando o entendimento do mecanismo de morte. Este é o $1^{\circ}$ estudo brasileiro a usar essa classificação, a qual foi utilizada com sucesso em dois estudos multicêntricos (AIRE e NETWORK) ${ }^{16}$.

Algumas limitações do estudo devem ser comentadas. Em primeiro lugar, nossas conclusões não devem ser estendidas para uma população geral com ICC. Estudos com pacientes não selecionados mostram mortalidade, ainda que elevada, menor que a por nós encontrada ${ }^{14}$. Além disso, incluímos pacientes com valvopatia e cardiomiopatia hipertrófica, freqüentemente excluídos em outros estudos ${ }^{19}$. Outra limitação é que não acompanhamos os pacientes desde sua $1^{a}$ internação por ICC, que seria o ideal para estudo de sobrevida. Finalmente, o cálculo da FE não foi feito pelo método ideal. O cálculo ecocardiográfico feito pelo método de Simpson apresenta maior acurácia que o empregado em nosso estudo ${ }^{30}$.

Os dados deste trabalho geraram a criação, em nosso hospital, de um comitê de melhoria da qualidade dos cuidados cardiovasculares, com a finalidade de educar o paciente e a família, ensinando-os a reconhecer sinais e sintomas que indiquem piora da ICC, e também com o intuito de aumentar a vigilância quanto à aderência ao tratamento, vacinação e pesquisa da etiologia da ICC, entre outros.

Concluindo, pacientes idosos com ICC atendidos em UE apresentam alta mortalidade intra-hospitalar e pobre prognóstico após a alta, a curto-prazo. $\mathrm{O}$ tratamento atual ainda é insatisfatório. A maioria morre por falência circulatória, decorrente da progressão da ICC. Maior vigilância e intervenções multidisciplinares poderiam melhorar a morbimortalidade e reduzir a taxa de reinternação.

\section{Referências}

1. Gibson TC, White KL, Klainer LM - The prevalence of congestive heart failure in two rural communities. J Chron Dis 1966; 19: 141-52.

2. Garrison GE, McDonough JR, Hames CG, Stulb SC - Prevalence of congestive heart failure in the population of Evans County, Georgia. Am J Epidemiol 1966; 83: 338-44.

3. Massie BM, Packer M-Congestive heart failure: current controversies and future prospects. Am J Cardiol 1990; 66: 429-30.

4. Eriksson H, Svärdsudd K, Larsson B et al - Dyspnoea in a cross-sectional and a longitudinal study of middle-aged men: the study of men born in 1913 and 1923. Eur Heart J 1987; 8: 1015-23.

5. Rich MW, Beckham V, Wittenberg C, Leven CL, Freedland KE, Carney RM - A multidisciplinary intervention to prevent the readmission of elderly patients with congestive heart failure. N Engl J Med 1995; 333: 1190-5.

6. National Center for Health Statistics. Graves EJ - 1989 Summary: national hospital discharge survey. Advance data from vital and health statistics. $\mathrm{N}^{\circ} 199$. Hyattsville. Md: Public Health Service. 1991: 1-12. (DHHS publication ${ }^{\circ}$ [PHS] 91-1250).

7. Massie BM, Packer M-Congestive heart failure: current controversies and future prospects. Am J Cardiol 1990; 66: 429-30.

8. Rocha N, Mesquita ET, Cardoso R, Gaspar S, Maia E, Kopiller E-Insuficiência cardíaca e o emprego de dobutamina na sala de emergência. Jornal Brasileiro Emergência 1996; VI: 15-16.

9. Pfeffer MA, Braunwald E, Moye LA et al - Effect of captopril on mortality and morbidity in patients with left ventricular dysfunction after myocardial infarction. N Engl J Med 1992; 327: 669.

10. The SOLVD investigators - Effect of enalapril on survival in patients with reduced left ventricular ejection fraction and congestive heart failure. NEngl J Med 1991; 325: 292-302.

11. The Acute Infarction Ramipril Efficacy (AIRE) Study Investigators - Effect of ramipril on mortality and morbidity of survivors of acute myocardial infarction with clinical evidence of heart failure. Lancet 1993; 342: 821-8.

12. Cohn JN, Johnson G, Ziesche S et al - Effect of vasodilator therapy on mortality in chronic congestive heart failure: results of a Veterans Administration Cooperative Study (V-HeFT I). N Engl J Med 1986; 314: 1547-52.

13. Andersson B, Waagstein F-Spectrum and outcome of congestive heart failure in a hospitalized population. Am Heart J 1993; 126: 632-40.

14. Ho KKL, Pinsky JL, Kannel WB, Levy D - The epidemiology of heart failure: The Framingham study. J Am Coll Cardiol 1993; 22: Suppl A: 6A-13A.

15. Carlson KJ, Lee DC-S, Goroll AH, Leahy M, Johnson RA - An analysis of physicians' reasons for prescribing long-term digitalis therapy in outpatients. J Chron Dis 1985; 38: 733-9.

16. NarangR,ClelandJGF,ErhardtLetal-Mode of deathinchronicheartfailure: a request and proposition for more accurate classification. Eur Heart J 1996; 17: 1390-403.
17. Ghali JK, Cooper R, Ford E-Trends in hospitalization rates for heart failure in the United States. 1973-1986: evidence for increasing population prevalence. Arch Intern Med 1990; 150: 769-73.

18. Franciosa JA, Wilen M,Ziesche S, Cohn JN - Survival in men with severe chronic left ventricular failure due to either coronary heart disease or idiopathic dilated cardiomyopathy. Am J Cardiol 1983; 51: 831-6.

19. Cohn JN, Levine TB, Olivari MT et al - Plasma norepinephrine as a guide to prognosis in patients with chronic congestive heart failure. N Engl J Med 1984; 311 : 819-23.

20. Brophy JM, Deslauriers G, Boucher B, Rouleau JL - The hospital course and short term prognosis of patients presenting to the emergency room with descompensated congestive heart failure. Can J Cardiol 1993; 9: 219-24.

21. Packer $\mathrm{M}$ - Do angiotensin-converting enzyme inhibitors prolong life in patients with heart failure treated in clinical practice? J Am Coll Cardiol 1996; 28 1323-7.

22. Rahimtoola SH-The hibernating myocardium in ischaemia and congestive heart failure. Eur Heart J 1993; 14(suppl A): 22-6.

23. Bonow RO - The hibernating myocardium: implications for management of congestive heart failure. Am J Cardiol 1995; 75: 17A-25A.

24. Weingarten SR, Riedinger MS, Shinbane J et al - Triage practice guideline for patients hospitalized with congestive heart failure: improving the effectiveness of the coronary care unit. Am J Med 1993; 94: 483-90.

25. Kornowski R, Zeeli D, Averbuch Met al - Intensive home-care surveillance prevents hospitalization and improves morbidity rates among elderly patients with severe congestive heart failure. Am Heart J 1995; 129: 762-6.

26. West JA, Miller NH, Parker KM et al - A comprehensive management system for heart failure improves clinical outcomes and reduces medical resource utilization. Am J Cardiol 1997; 79: 58-63.

27. Domanski MJ, Garg R, Yusuf S - Prognosis in congestive heart failure. In: Hosenpud JD, Greenberg BH, eds - Congestive Heart Failure: Pathophysiology, Diagnosis, and Comprehensive Approach to Management. New York: SpringerVerlag, 1994: 622-7.

28. Lee W, Packer M-Prognostic significance of serum sodium concentration and its modifications by converting enzyme inhibition in patients with severe chronic heart failure. Circulation 1986; 73: 257-67.

29. Hermann DD, Greenberg BH - Prognostic factors. In: Poole-Wilson PA, Colucci WS, Massie BM, Chattenjee K, Coats AJS, eds - Heart Failure. New York: Churchill Livingstone, 1997: 439-54.

30. Folland ED, Parisi AF, Moynihan PF et al - Assessment of left ventricular ejection fraction and volumes by real time two dimensional echocardiography: a comparison of cineangiographic and radionuclide techniques. Circulation 1979; 60: 760-6. 